\title{
Intestinal and hepatic drug transporters: pharmacokinetic, pathophysiological, and pharmacogenetic roles
}

\author{
Tomohiro Terada $\cdot$ Daiki Hira
}

Received: 24 February 2015/ Accepted: 24 February 2015/Published online: 14 March 2015

(C) Springer Japan 2015

\begin{abstract}
The efficacy and safety of pharmacotherapies are determined by the complex processes involved in the interactions between drugs with the human body, including pharmacokinetic aspects. Among pharmacokinetic factors, it has been recognized that drug transporters play critical roles for absorption, distribution and excretion of drugs, regulating the membrane transport of drugs. The vast amounts of information on drug transporters collected in the past 20 years have been organized according to biochemical, molecular, genetic, and clinical analyses. Novel technologies, public databases, and regulatory guidelines have advanced the use of such information in drug development and clinical practice. In this review, we selected some clinically important drug transporters expressed in the intestine and liver, and introduced the research history and current knowledge of their pharmacokinetic, pathophysiological, and pharmacogenetic implications.
\end{abstract}

Keywords Drug transporters · Pharmacokinetics ·

Polymorphisms · Drug-drug interaction

$\begin{array}{ll}\text { Abbreviations } \\ \text { PK } & \text { Pharmacokinetics } \\ \text { PD } & \text { Pharmacodynamics } \\ \text { CYP } & \text { Cytochrome P450 } \\ \text { ABC } & \text { ATP-binding cassette } \\ \text { P-gp } & \text { P-glycoprotein }\end{array}$

This invited article was registered at the editorial office on behalf of the authors.

T. Terada $(\bowtie) \cdot$ D. Hira

Department of Pharmacy, Shiga University of Medical Science

Hospital, Otsu, Shiga 520-2192, Japan

e-mail: teradat@belle.shiga-med.ac.jp
HGNC Human gene nomenclature committee

SLC Solute carriers

ITC International transporter consortium

FDA Food and drug administration

EMA European medicines agency

PMDA Pharmaceuticals medical devices agency

BCRP Breast cancer resistance protein

OATP Organic anion transporting polypeptide

OCT Organic cation transporter

OAT Organic anion transporter

MATE Multidrug and toxin extrusion

BSEP Bile salt export pump

MRP Multidrug resistance proteins

PEPT Peptide transporter

IBD Inflammatory bowel disease

PFIC2 Progressive familial intrahepatic cholestasis type 2

DILI Drug-induced liver injury

SNP Single nucleotide polymorphism

AUC Area under the curve

\section{Research history on drug transporters}

Drug efficacy and safety are determined by the interplay of multiple processes that regulate pharmacokinetics (PK) (absorption, distribution, metabolism, and excretion) and pharmacodynamics (PD) (drug action). The pharmacological effects of orally administered drugs are dependent on their adequate intestinal absorption and distribution before being eliminated via metabolic and excretory pathways. Although drug-metabolizing enzymes such as cytochrome P450 (CYP) were previously considered to be the key determinants of pharmacokinetics, the membrane transport processes mediated by drug transporters are now 
recognized as being important for pharmacokinetic properties (Fig. 1) [1].

Studies on membrane vesicles and cultured epithelial cell lines were introduced into the research field of drug transport in the early 1980s, and the biochemical characterization of drug transport has since advanced. Drug transporters are functionally classified as primary and secondary active transporters. Primary active transporters include ATP-binding cassette (ABC) transporters that use the hydrolysis of ATP as a driving force, while secondary active transporters use various driving forces such as extracellular ion gradients and membrane potentials, according to the physicochemical properties of substrates and membrane localization of transporters.

The molecular nature of drug transporters was clarified by the end of the $1980 \mathrm{~s}$, with the first clinically important drug transporter, the $\mathrm{P}$-glycoprotein (P-gp), being identified in 1986 [2]. In the 1990s, significant advances occurred in the molecular identification of many drug transporters, leading to a deeper understanding of their genetic history and grouping. In the 2000s, the Human Gene Nomenclature Committee (HGNC) classified transporters (not limited to drug transporters) based on sequence similarities as $\mathrm{ABC}$ transporters [3] and solute carriers (SLCs) [4]. For example, P-gp was named as ABCB1. More than 400 membrane transporters have now been annotated in the human genome, and many members of the ABC and SLC family function as drug transporters.

In 2007, the International Transporter Consortium (ITC), consisting of industrial, regulatory, and academic scientists, was formed to obtain a clearer understanding of the role of drug transporters in drug development and clinical pharmacology. The ITC published its first report in 2010 [5], which provided (1) an overview of key transporters; (2) an evaluation of transporter-related drug-drug interactions; and (3) criteria for the design and conduct of clinical studies on transporter-related drug interactions. Regulatory agencies such as the U.S. Food and Drug Administration (FDA) subsequently published guidance documents on drug transporters and drug interactions based on this report [6]. The ITC continued to discuss additional and clinically relevant transporters and provide regulatory guidance, and published its second report in 2013 [7]. Regulatory agencies such as the European Medicines Agency (EMA) and Pharmaceuticals Medical Devices Agency (PMDA) in Japan also published guidelines for drug interaction studies on transporters [8, 9].

Seven of the following drug transporters were selected as clinically important drug transporters in the first report of the ITC: (1) P-glycoprotein (P-gp, also named as ABCB1); (2) breast cancer resistance protein (BCRP, ABCG2 as the HGNC name); (3) organic anion-transporting polypeptide (OATP)1B1 (SLCOB1 as the HGNC name); (4) OATP1B3 (SLCOB3 as the HGNC name); (5) organic cation transporter (OCT)2 (SLC22A2 as the HGNC name); (6) organic anion transporter (OAT)1 (SLC22A6 as the HGNC name); and (7) OAT3 (SLC22A8 as the HGNC name). In its second report, the following were added as clinically important drug transporters: (8) multidrug and toxin extrusion (MATE)1/MATE2 K (SLC47A1/SLC47A2 as the HGNC name); (9) bile salt export pump (BSEP) (ABCB11 as the HGNC name); (10)

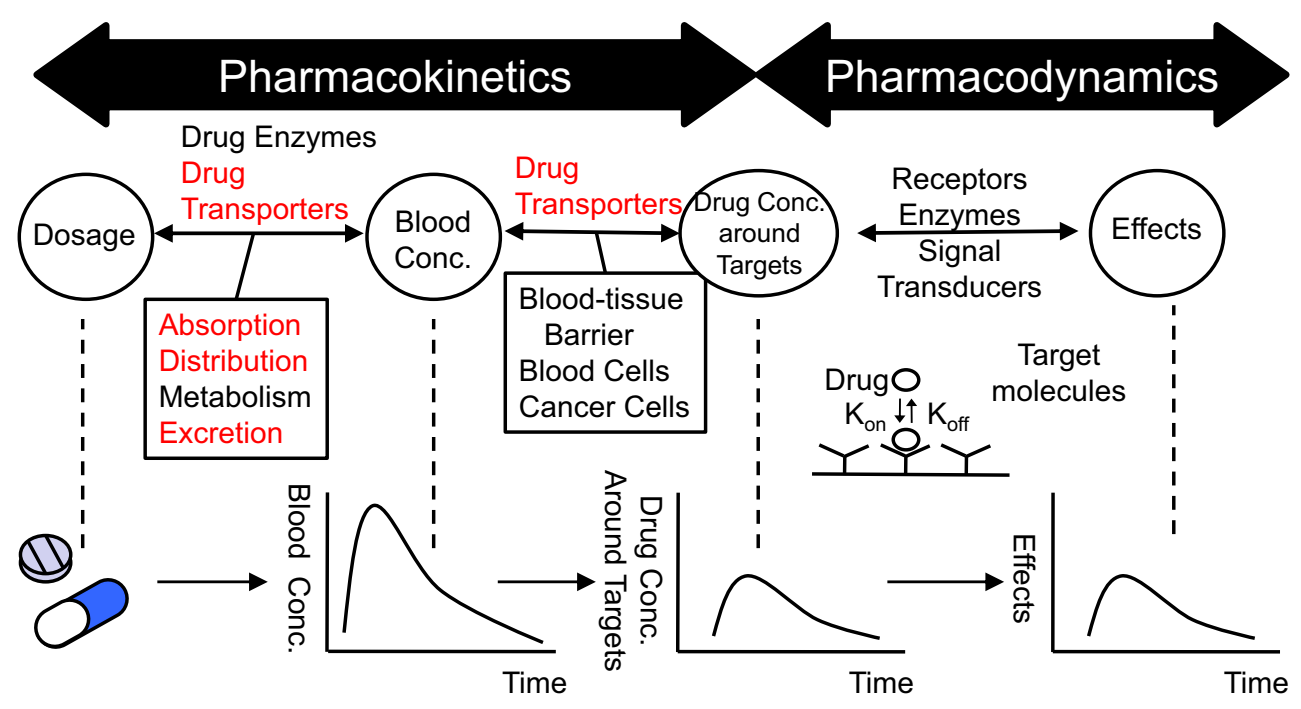

Fig. 1 PK/PD relationship and drug transporters. Drug transporters play critical roles for absorption, distribution and excretion of drugs with regulating the membrane transport of drugs. Thus, accompanied with drug-metabolizing enzymes, drug transporters determine the drug exposure to the body. Furthermore, this protein also regulates the drug concentration (conc.) around target sites by the uptake or efflux of substrate drugs. These balances of total and/or local drug concentration may influence in part the side effects and/or pharmacological effects of drugs 
multidrug resistance proteins (MRPs: especially MRP2, MRP3, MRP4, ABCC2 ABCC4 as the HGNC name); (11) equilibrative nucleoside transporter 1; and (12) peptide transporter (PEPT)1 (SLC15A1 as the HGNC name). P-gp, BCRP, BSEP, and MRPs are members of the ABC transporter family, while the others belong to the SLC family. The characteristics and tissue distribution of these drug transporters were summarized in Table 1 and Fig. 2.

Of these 12 drug transporters, the following drug transporters, which are mainly expressed in the intestine and liver, were focused on in this review, and a general description and current clinical topics were introduced. The selected transporters were as follows: ABC transporters (Pgp, BCRP, BSEP, and MRPs) and SLC drug transporters (OATP1B1, OAPT1B3, and PEPT1). Furthermore, the part of the second report of the ITC referred to two clinically important polymorphisms in OATP1B1 (SCLO1B1 c.521T > C, p.V174A) and BCRP (ABCG2 c.421C > A, p.Q141 K), and we also herein discussed the clinical relevance of these polymorphisms.

\section{ABC transporters}

\section{MDR1 (P-glycoprotein, P-gp; ABCB1)}

P-glycoprotein (P-gp; ABCB1) was originally isolated from cancer cells, in which it was shown to extrude chemotherapeutic agents out of the cell, thereby conferring multidrug resistance [2]. A subsequent study reported that P-gp was also expressed in on the luminal sides of various normal tissues, and was involved in the transport of various drugs, mediating the efflux of drugs from the intracellular to extracellular space [10]. In vivo studies using genedisrupted animal models of this transporter demonstrated that P-gp played important physiological and pharmacokinetic roles in protecting the body against xenobiotics; namely, regulating oral bioavailability and the hepatic/renal excretion of substrates, as well as forming part of the blood-tissue barrier [3]. The gatekeeper function of P-gp in the gut is desirable for toxins and carcinogens, but was also found to limit the oral availability of clinically important drugs. Individual differences in the activity and/or expression of the protein were shown to induce changes in drug bioavailability $[11,12]$.

P-gp has extremely broad substrate specificity, with a preference towards lipophilic, cationic compounds, and the list of its substrates/inhibitors includes anticancer agents, antivirals, calcium channel blockers, immunosuppressive agents, and digoxin. As these drugs have a narrow therapeutic range, there are many examples for clinically relevant drug interactions. Representative examples were described in Table 1. Since an estimation of the impact of
P-gp on human pharmacokinetics is critical for drug development, drug interaction guidelines have provided a decision tree to determine whether the drugs being investigated can function as P-gp inhibitors in order to avoid transporter-related drug-drug interactions and inter-individual variability.

Regarding pharmacogenomics, many association studies have been conducted between popular variants of the $A B C B 1$ gene $\quad($ c. $3435 \mathrm{C}>\mathrm{T}, \quad$ c. $2677 \mathrm{G}>\mathrm{T} / \mathrm{A}, \quad$ and c. $1236 \mathrm{C}>\mathrm{T}$ ) and the pharmacokinetics and/or pharmacodynamics of P-gp substrates since 2000 [13]. However, in many cases, the reported effects of $A B C B 1$ gene variants (especially c. $3435 \mathrm{C}>\mathrm{T}$ ) have been inconsistent and, in some cases, conflicting. A meta-analysis on the influence of the $A B C B 1$ c. $3435 \mathrm{C}>\mathrm{T}$ polymorphism on digoxin (the typical substrate of $\mathrm{P}$-gp) pharmacokinetics revealed that this polymorphism did not affect the pharmacokinetics of digoxin or expression of ABCB1 mRNA [14]. Taken together, these findings indicated that the routine application of an $A B C B 1$ gene polymorphism analysis to clinical studies was not warranted at this time.

Investigations on polymorphisms in the $A B C B 1$ gene have been performed not only for pharmacotherapies, but also for the etiology and prognosis of various disorders [13]. To date, the following diseases have been examined: Parkinson's disease, epilepsy, depression, systemic lupus erythematosus, inflammatory bowel disease (IBD), cancers (leukemia, colon cancer, renal cell carcinoma, and glioma), liver and renal diseases (cirrhosis and nephritic syndrome), gingival hyperplasia, rheumatoid arthritis, and hypertension. However, definitive conclusions have not yet been reached for most of these diseases. For example, a previous study reported that variants in the $A B C B 1$ gene were associated with the development of IBD [15], but a meta-analysis did not provide any sufficient evidence to deny or confirm an association for all variants with IBD [16].

\section{BCRP (ABCG2)}

BCRP was also originally identified as a determinant of multidrug resistance in cancer cell lines in vitro [17]. Subsequent studies demonstrated that BCRP was expressed in normal tissues, such as the gastrointestinal tract, liver, kidney, brain endothelium, testis, and placenta, and localized to apical membranes. BCRP is often expressed in stem cell populations. Unlike other ABC transporters, BCRP only has one ATP-binding cassette and six putative transmembrane domains, and thus, is referred to as a half- $\mathrm{ABC}$ transporter, most likely functioning as a homodimer. In contrast to structural differences, BCRP shows similar biochemical characteristics to those of other ABC transporters; it pumps out the substrate, xenobiotics, using ATP 


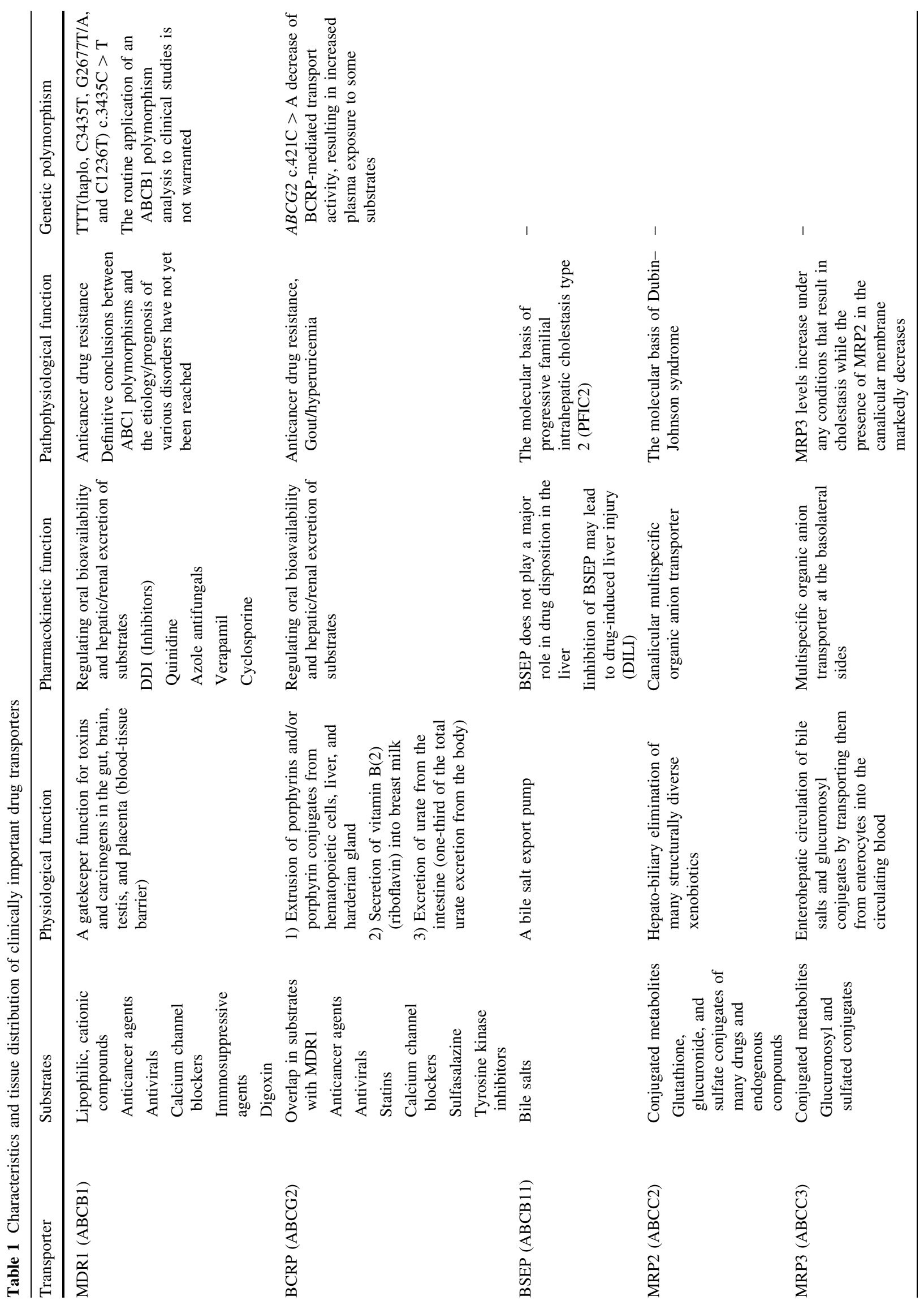




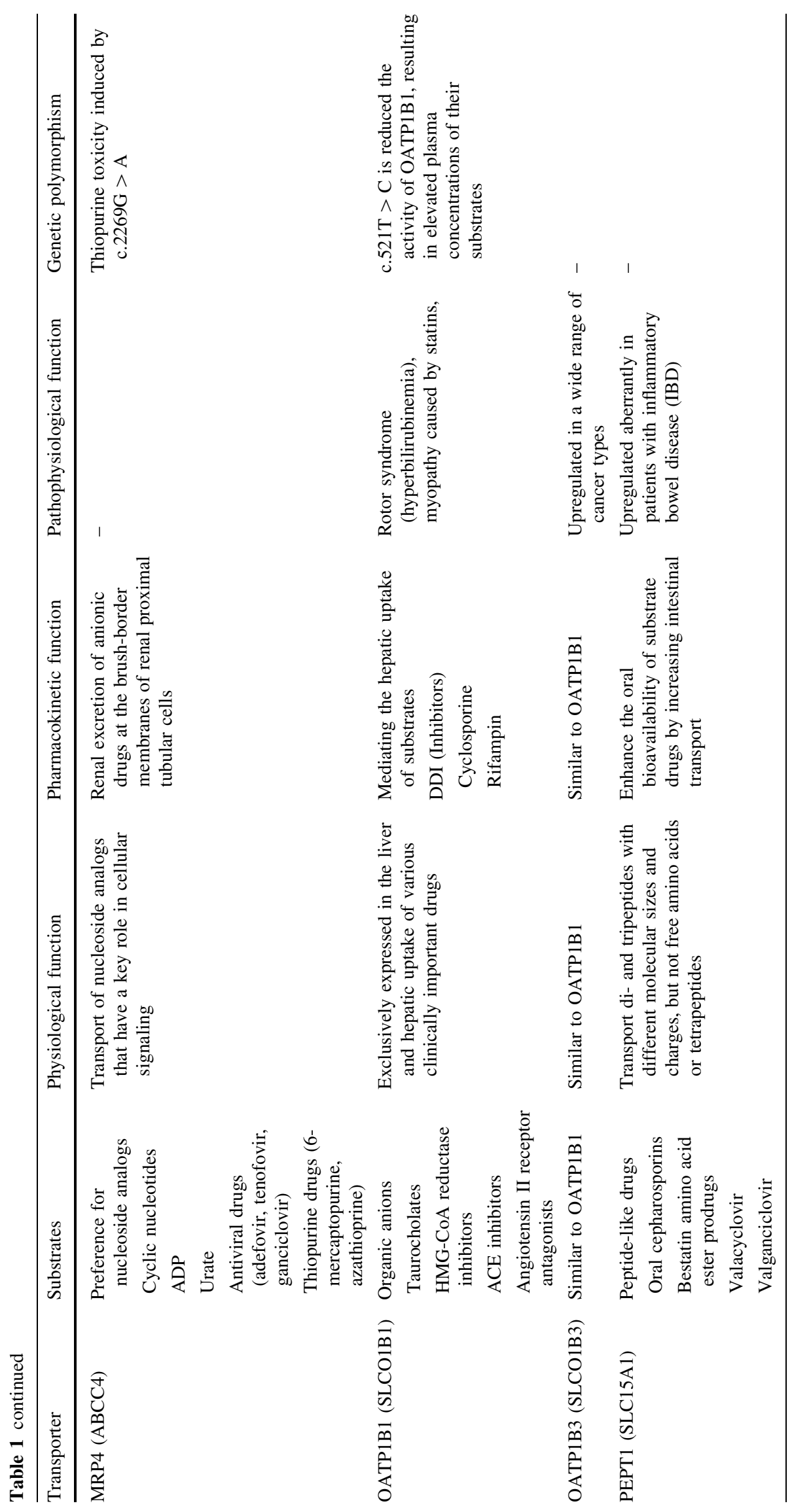


(a) Intestinal epithelia

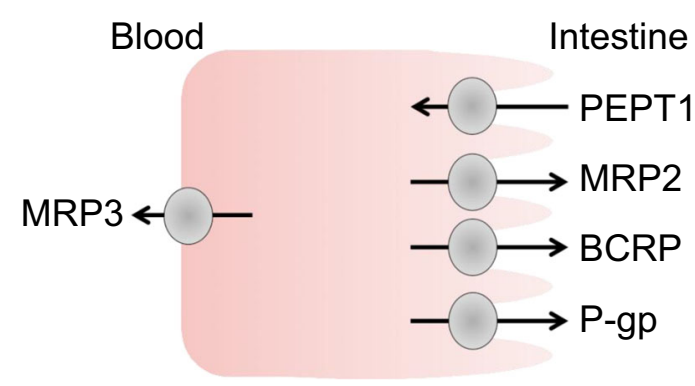

(c) Kidney proximal tubule

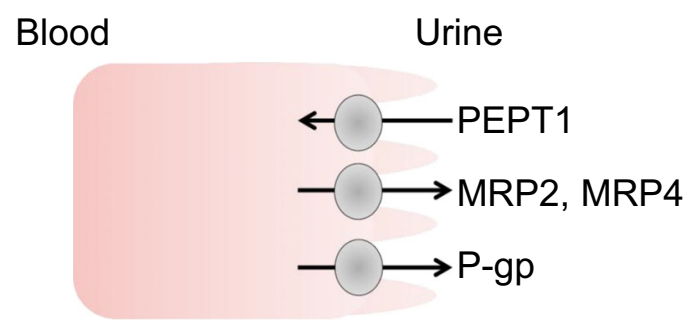

(b) Human hepatocyte

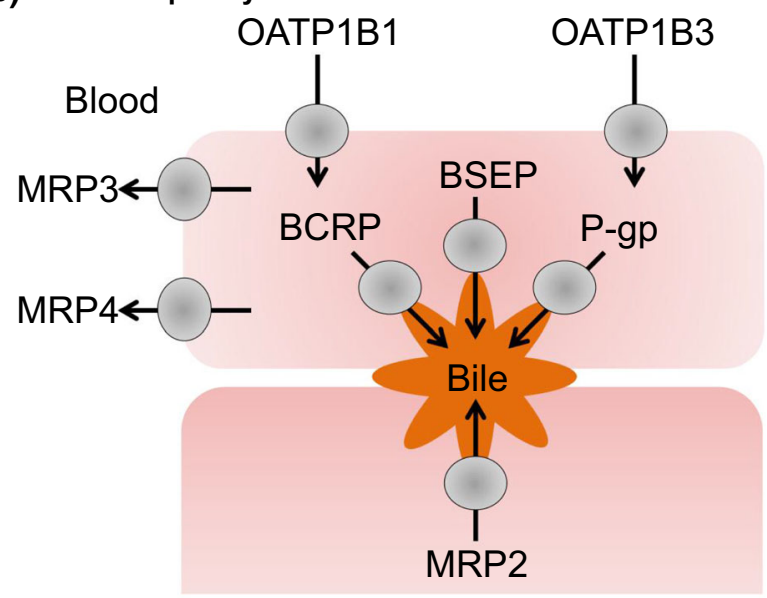

(d) Blood-brain barrier

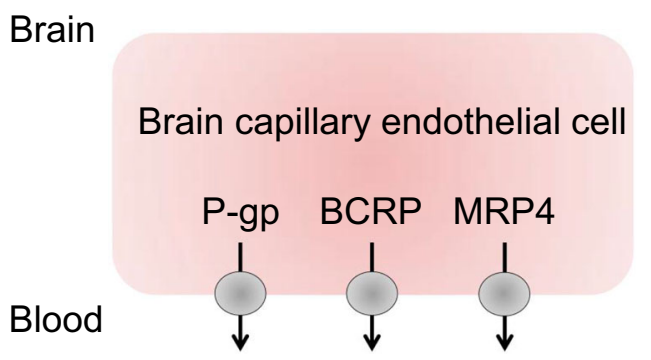

in the hepatocyte basolateral membrane include MRP3 and MRP4 (ABCC4). Canalicular efflux pumps in hepatocytes comprise P-gp, BSEP (ABCB11), BCRP (ABCG2), and MRP2 (ABCC2). c Kidney proximal tubules contain PEPT1; MRP2 and MRP4; P-gp in the apical (luminal) membrane. d Apical (luminal) transport proteins in brain capillary endothelial cells that contribute to the function of the blood-brain barrier include the efflux pumps P-gp, BCRP, and MRP4

chemotherapy drugs including antivirals, statins, antibiotics, calcium channel blockers, and sulfasalazine [23]. Since 2005, the focus of research has shifted to tyrosine kinase inhibitors, such as imatinib, erlotinib, and sunitinib, for cancer pharmacotherapy. Similar to P-gp, regulatory guidelines have recommended assessments to determine whether a drug candidate is a substrate and/or an inhibitor of BCRP.

Regarding pharmacogenomics, a large number of association studies between $A B C G 2$ gene polymorphisms and the $\mathrm{PK} / \mathrm{PD}$ of substrate drugs have been performed to date, and have focused on the $A B C G 2$ c. $421 \mathrm{C}>\mathrm{A}, \mathrm{p} . \mathrm{Q} 141 \mathrm{~K}$ variant. These clinical topics will be discussed in Sect. 5.2. The nonsynonymous single nucleotide polymorphism (SNP), c.421C > A (p.Q141 K), which is localized in the ATP-binding domain, has been associated with impaired activity in vitro [24]. In addition to pharmacotherapy, c. $421 \mathrm{C}>\mathrm{A}$ has been implicated in elevated uric acid levels and gout/hyperuricemia risk [25, 26]. 


\section{BSEP (ABCB11)}

One of the primary roles of the liver is to produce and secrete bile acid in order to solubilize and absorb dietary lipids. The bile salt export pump (BSEP, ABCB11) mediates the ATP-dependent efflux of monovalent bile salts across canalicular membranes. This transporter constitutes a rate-limiting step in the transport of bile salts from the blood into the bile and thereby acts as an important determinant of bile flow [27]. Thus, dysfunction of BSEP may induce the intrahepatic cholestasis, i.e., a mutation in the BSEP gene such as the progressive familial intrahepatic cholestasis type 2 (PFIC2) or by acquired factors such as the inhibition of BSEP by administered drugs [28].

Although BSEP does not play a major role in drug disposition in the liver, the inhibition of BSEP may lead to the intracellular accumulation of bile salts followed by cytotoxic events including drug-induced liver injury (DILI) as mentioned above. DILI has been the most frequent cause of safety-related drug marketing withdrawals in the United States during the last decade [29]. In addition to a reduction in the quality of life of individual patients and costs of associated health care, DILI poses a major economic challenge to the pharmaceutical industry because of decreasing marketing approval rates, post-marketing restrictions, and boxed warnings. These concepts promoted to suggest that BSEP is categorized as a clinically important drug transporter [30]. Difficulties are currently associated with predicting significant BSEP-mediated DILI; therefore, prospective BSEP testing cannot be endorsed. However, the in vitro characterization of BSEP-drug interactions is certainly warranted following the appearance of cholestatic issues in clinical trials or safety studies. The EMA drugdrug interaction guideline states that BSEP inhibitory potentials need to be considered for investigation. If inhibition is indicated, adequate biochemical monitoring including serum bile salts is recommended during drug development.

\section{MRPs (ABCCs)}

Although the MRP family consists of nine members, MRP2 (ABCC2), MRP3 (ABCC3), and MRP4 (ABCC4) have received particular attention because of their roles in the disposition of drugs and conjugates [31]. An overlap has been reported in substrate specificity between MRP members, whereas differences have been detected in tissue distribution and membrane localization. Regarding substrate preferences, lipophilic anions can be accepted by MRPs, and these characteristics are in contrast to the preference for lipophilic cations by P-gp.
MRP2 (ABCC2)

MRP2 was functionally characterized as a canalicular multispecific organic anion transporter, i.e., cMOAT. The molecular identification and characterization of this transporter demonstrated that MRP2 played a major role in the hepato-biliary elimination of many structurally diverse xenobiotics [32]. This transporter can accept a diverse range of substrates, including glutathione, glucuronide, and the sulfate conjugates of many drugs and endogenous compounds. The arachidonic acid-derived glutathione conjugate termed leukotriene $\mathrm{C} 4$, as well as glucuronic acid conjugates, including bilirubin monoglucuronide and bilirubin bisglucuronide are also transported by MRP2 [33].

Although a large number of genetic variants have been identified in the $A B C C 2$ gene, only some lead to functional impairments in MRP2 [33]. Inactive MRP2 has been recognized as the molecular basis of Dubin-Johnson syndrome, which is associated with mild, predominantly conjugated hyperbilirubinemia [34]. Under this condition, alternative efflux pathways, including basolateral hepatocellular MRP3 and apical MRP4 in proximal tubule cells in the kidney, compensate for the impairment in MRP2 function through the efflux of conjugated bilirubin and other endogenous and xenobiotic anionic substrates into the blood and urine, respectively [35].

\section{MRP3 (ABCC3)}

MRP3 shows approximately $50 \%$ amino acid identity to MRP2. In contrast to other ABC drug transporters, MRP3 is localized in the basolateral membranes of epithelial cells in the liver and intestine [36]. Substrates for MRP3 include glucuronosyl and sulfated conjugates, whereas glutathione conjugates are poorer substrates for MRP3 than for MRP1 and MRP2 [37]. Since MRP3 also transports some bile salts [38], it has been suggested to play important roles in the enterohepatic circulation of bile salts by transporting them from enterocytes into the circulating blood. As described above, MRP3 levels are known to increase under any conditions that result in cholestasis, whereas MRP2 levels in the canalicular membrane markedly decrease [39]. These findings suggest the existence of a reciprocal relationship between MRP2 and MRP3, which can protect the liver from the accumulation of potentially toxic bile constituents [40].

Although multiple genetic polymorphisms exist in the $A B C C 3$ gene, no major PK consequences have been documented to date. Mice lacking Mrp3 are viable and fertile and have no apparent phenotype [41]. 


\section{MRP4 (ABCC4)}

MRP4 lacks an additional N-terminal five-transmembrane domain, which is characteristic of longer MRPs such as MRP2 and MRP3 [40]. This transporter shows unique membrane localization in polarized cells. MRP4 is localized to the basolateral membranes in hepatocytes, but is expressed in the apical membranes in renal proximal tubule cells. MRP4 was found to be localized to the apical and basolateral membranes in colonic epithelial cells, with a higher apical abundance [42].

Substrates for MRP4 include endogenous molecules that have a key role in cellular signaling, including cyclic nucleotides, adenosine diphosphate, eicosanoids, urate, and conjugated steroid hormones [43]. Other potentially relevant physiological substrates are folate, bile acids, and glutathione, which is co-transported with bile acids [44]. As a drug transporter, MRP4 shows broad substrate specificity covering antiviral (adefovir, tenofovir, and ganciclovir), antibiotic (cephalosporins), cardiovascular (loop diuretics, thiazides, and angiotensin II receptor antagonists), and cytotoxic (methotrexate, 6-thioguanine, 6-mercaptopurine, and topotecan) agents.

The ABCC4 gene is highly polymorphic; large variabilities in the expression of ABCC4/MRP4 mRNA (38fold) and protein (45-fold) were found in the human liver, and these expression levels were previously reported to be significantly upregulated in the livers of patients with cholestasis [45]. A common SNP (c.2269G > A; > $18 \%$ in the Japanese population) that reduces MRP4 function appears to be associated with thiopurine toxicity [46, 47].

\section{SLC drug transporters}

\section{OATPs (SLCOs)}

In 1994, a $\mathrm{Na}^{+}$-independent organic anion-transporting polypeptide (oatp1) was originally cloned from a rat liver cDNA library [48]. Thereafter, many isoforms of OATP were identified in various species; however, unlike other transporters, this family exhibits large inter-species differences [49]. HGNC designated the OATP family as the SLC21 family, whereas a traditional SLC21 classification does not permit an unequivocal and species-independent identification of each isoform; therefore, all members are newly classified within the SLCO superfamily. The methods of classification and nomenclature were previously described in detail [49].

All OATPs contain 12 transmembrane domains. Certain transporters show a more restricted tissue expression pattern (i.e., SLCO1B1 and SLCO1B3/liver), while others such as SLCO2B1 can be detected in almost every tissue that has been investigated to date [50]. This finding indicates that some OATPs have organ-specific functions while others may be involved in housekeeping functions.

OATP families mediate the $\mathrm{Na}^{+}$-independent transport of a wide range of amphipathic organic compounds including bile salts, organic dyes, steroid conjugates, thyroid hormones, anionic oligopeptides, numerous drugs, and other xenobiotic substances [49].

\section{OATP1B1 (SLCO1B1)}

OATP1B1 has been well characterized among the human OATP family. This transporter is exclusively expressed in the liver and located on sinusoidal membranes. Thus, the major pharmacokinetic role of OATP1B1 is the hepatic uptake of various clinically important drugs such as methotrexate, statins, and angiotensin II receptor antagonists [51].

Several clinically used drugs such as cyclosporine (an immunosuppressant) and rifampin (antituberculosis drug) potently inhibit OATP1B1, causing clinically significant drug interactions. For example, cyclosporine was shown to elevate the AUC of simvastatin by approximately eightfold, whereas no significant changes were observed in simvastatin levels when it was coadministered with tacrolimus [52]. Since many inhibitors are not OATP1B1 substrates, predicting this interaction seems to be difficult, and therefore, regulatory guidelines have recommended determining whether a drug candidate is a substrate and/or inhibitor of OATP1B1. The potential risks of drug-drug interactions between statins and candidate drugs have already been described in detail [53].

Regarding pharmacogenomics, a large number of association studies between OATP1B1 polymorphisms and the $\mathrm{PK} / \mathrm{PD}$ of substrate drugs have been performed to date, with a particular focus on the OATP1B1 c.521T $>\mathrm{C}$, p.V174A variant. These clinical topics will be discussed in Sect. 5.1. The nonsynonymous SNP, c.521T $>$ C (p.V174A), resulted in a decrease in the membrane expression of OATP1B1 and decreased transport activity [54].

\section{OATP1B3 (SLCO1B3)}

Similar to OATP1B1, the expression of OATP1B3 is limited to the sinusoidal membranes of hepatocytes, and this transporter shares most of its substrates and inhibitors with OATP1B1, suggesting that the pharmacokinetic roles of both transporters are very similar. The contribution of each transporter for drug disposition has not yet been ascertained; however, OATP1B1 may be a major transporter. Clinically relevant genetic polymorphisms in OATP1B3 have so far not been reported. 
The pathophysiological implication of both transporters, especially in cancer, appears to be different. The expression of OATP1B1 and OATP1B3 is generally reduced in hepatocellular carcinomas, whereas that of OATP1B3 is upregulated in a wide range of cancer types. OATP1B3 can transport anticancer drugs as well as hormones and hormone precursors, all of which can affect the growth and survival of cancer cells [55]. These findings suggest that OATP1B3 plays a significant role in cancer progression or responses to treatments.

\section{PEPT1 (SLC15A1)}

PEPT1 was initially identified by expression cloning, and PEPT2, the isoform of PEPT1, was subsequently isolated. Both transporters showed approximately $50 \%$ amino acid identity [56]. PEPT1 is localized to the brush-border membranes of intestinal and renal epithelial cells, whereas PEPT2 is preferentially expressed in the kidney and located in the brush-border membranes of renal epithelial cells.

PEPT1 can accept di- and tripeptides as physiological substrates, indicating that the substrate specificity of this transporter is markedly broader than those of other nutritional transporters. Accordingly, foreign compounds structurally resembling small peptides such as oral $\beta$-lactam antibiotics were shown to be recognized by PEPT1, and thus, this transporter functions as a drug transporter [56]. Over the last two decades, PEPT1 has been used as a target to improve the intestinal absorption of poorly absorbed drugs through amino acid-based modifications. For example, the enhanced oral bioavailability of valacyclovir and valganciclovir, L-valine ester prodrugs of acyclovir and ganciclovir, respectively, has been attributed to their enhanced intestinal transport via PEPT1 $[57,58]$. To date, no clinical consequences have been identified for any PEPT1 variants.

Regarding the pathophysiological implications of PEPT1, extensive evidence has been accumulated to support the role of PEPT1 in IBD. Although PEPT1 is weakly expressed in the large intestine, it is aberrantly upregulated in patients with IBD $[59,60]$. In vitro transport studies demonstrated that PEPT1 transported bacteria derived small peptides such as $\mathrm{N}$-formyl-methionyl-leucyl-phenylalanine, and these small peptides interacted with NOD-like receptors to trigger the nuclear factor-KB and MAPK pathways [61-63]. These reactions have been hypothesized to induce the downstream production of proinflammatory cytokines/chemokines as well as the subsequent migration of neutrophils into regions of inflammation, and previous studies using human PEPT1 transgenic mice confirmed these reactions [64].

\section{Clinically important polymorphisms of drug transporters}

\section{OATP1B1 (SCLO1B1) c.521T > C, p.V174A}

Some haplotypes consist of combinations of SNPs. The activities of SLCO1B1*5 (c.521T > C, p.V174A) and *15 (c.388A > G, p.N130D and c.521T > C, p.V174A) were reduced, whereas those of $* 1 b(c .388 \mathrm{~A}>\mathrm{G}, \mathrm{p} . \mathrm{N} 130 \mathrm{D})$ and *14 (c.388A > G, p.N130D and c.463C > A, p.P155T) were increased. The allele frequency of SLCO1B1 gene was also markedly different [65]. Among the many polymorphisms in SLCO1B1 gene, c.521T > C has been identified as a clinically important polymorphism. This SNP resulted in a decrease in the membrane expression of OATP1B1 and decreased transport activity. Therefore, the hepatic uptake of OATP1B1 substrate drugs may be reduced and its plasma concentration elevated during pharmacotherapy, which will induce severe side effects in some cases. For example, the plasma area under the curve (AUC) of simvastatin acid was approximately threefold higher in those with the c.521CC genotype than in those with the c.521TT genotype [66]. A genome-wide association study showed that the SLCO1B1 c.521C allele increased the risk of myopathy with an odds ratio of 4.5 per copy of the C allele [67]. In the drug-drug interaction between cyclosporine and repaglinide, cyclosporine inhibited the CYP3A4-mediated metabolism and OATP1B1-mediated hepatic uptake of repaglinide. The effect of cyclosporine on the repaglinide AUC was $42 \%$ less in subjects with the SLCO1B1 521TC genotype than in those with the 521TT genotype [68]. In addition, a genome-wide association study demonstrated that $S L C O 1 B 1$ genetic variations were important determinants of the pharmacokinetics and clinical effects of methotrexate [69]. The pharmacokinetics of the HIV drugs, lopinavir and ritonavir, also varied with SLCO1B1 c.521T > C [70, 71].

\section{BCRP (ABCG2) c.421C > A, p.Q141 K}

The major nonsynonymous variant, $A B C G 2$ c. $421 \mathrm{C}>\mathrm{A}$, p.Q141 K, is known as a clinically important polymorphism in the PK/PD of their substrates, and is frequently observed in Asian rather than Caucasian or African American populations. The racial variation of allelic frequency resulted in a relatively high frequency in Asians, leading to a wide inter-individual variability in the PK of BCRP substrates. Clinical studies showed that the pharmacokinetics of sulfasalazine [72-74], rosuvastatin [7578], and other statins [79] in patients with $A B C G 2$ c. $421 \mathrm{C}>\mathrm{A}$ varied due to decreases in BCRP-mediated transport activity. The area under the plasma concentra- 
tion-time curve (AUC) and maximum plasma concentration $(\mathrm{Cmax})$ of rosuvastatin after the oral (p.o.) administration to $A B C G 2$ c.421 AA subjects were approximately 2.4-fold higher than those in $A B C G 2$ c. 421 CC subjects, whereas the time to reach $\mathrm{Cmax}$ (tmax) and the elimination terminal half-life remained unchanged, suggesting that SNP affected the intestinal absorption of rosuvastatin. In addition to statins, the clinical importance of $A B C G 2$ polymorphisms on PK/PD of tyrosine kinase inhibitors is now under intense investigation. Takahashi et al. reported that the recommended dosage of imatinib, a Bcr-Abl tyrosine kinase inhibitor for the treatment of chronic myelogenous leukemia, should be $400 \mathrm{mg}$ for patients with the $A B C G 2421 C C$ genotype, and $300 \mathrm{mg}$ for patients with the $421 \mathrm{CA}$ or 421AA genotype in order to attain a plasma threshold of approximately $1000 \mathrm{ng} / \mathrm{ml}$ [80]. Regarding gefitinib, an EGFR tyrosine kinase inhibitor, $A B C G 2$ c. $421 \mathrm{C}>\mathrm{A}$ was associated with the development of diarrhea in 124 patients treated with oral gefitinib $250 \mathrm{mg}$ once daily; seven (44\%) out of 16 patients heterozygous for $A B C G 2$ c. $421 \mathrm{C}>$ A developed diarrhea in contrast to only $13(12 \%)$ out of 108 patients homozygous for the wildtype sequence [81]. The pharmacokinetics and side effects of erlotinib, the tyrosine kinase inhibitor, and its major metabolite, OSI-420, have also related been related to $A B C G 2$ c.421C $>$ A $[82,83]$. Mizuno et al. [84, 85] reported that the dose-adjusted AUC of sunitinib, a multitargeted receptor tyrosine kinase inhibitor, was significantly higher in patients with a heterozygous variant for ABCG2 c. $421 \mathrm{C}>\mathrm{A}$ than in wild-type patients. A population PK analysis showed that the plasma concentration of sunitinib at a dosage of $25 \mathrm{mg}$ in $A B C G 2$ c.421CA and AA patients was similar to that at a dosage of $50 \mathrm{mg}$ in wild-type patients [86].

\section{Conclusions and perspectives}

We herein outlined the general characteristics of major intestinal and hepatic drug transporters and their clinical implications. Molecular information on each transporter has been organized over the past 20 years, and novel technologies and various useful public databases and guidelines have improved our understanding of the physiological and pharmacotherapeutic roles of drug transporters. Actually, these research outcomes were applied to drug development and clinical practices, and severe drug transporter-mediated drug interaction and DILI could be avoidable beforehand. The second ITC reports said that continual collaborations among stakeholdersindustry, global regulatory agencies, academia, and others-will be important to advance the understanding of the role of transporters in drug development and clinical pharmacology [7], and we hope such integration will contribute to more useful clinical outcomes.

Acknowledgments Work in the authors' laboratory is supported by a Grant-in-Aid for Scientific Research from the Japanese Ministry of Education, Culture, Sports, Science, and Technology.

Conflict of interest The authors declare that they have no conflict of interest.

\section{References}

1. Giacomini KM, Sugiyama Y. Membrane transporters and drug response. Brunton LL, Chabner BC, Knollman BA, editors. New York: McGraw-Hill Education; 2010.

2. Roninson IB, Chin JE, Choi KG, et al. Isolation of human mdr DNA sequences amplified in multidrug-resistant $\mathrm{KB}$ carcinoma cells. Proc Natl Acad Sci USA. 1986;83:4538-42.

3. Schinkel AH, Jonker JW. Mammalian drug efflux transporters of the ATP binding cassette (ABC) family: an overview. Adv Drug Deliv Rev. 2003;55:3-29.

4. Hediger MA, Romero MF, Peng JB, et al. The ABCs of solute carriers: physiological, pathological and therapeutic implications of human membrane transport proteins introduction. Pflugers Arch. 2004;447:465-8.

5. Giacomini KM, Huang SM, Tweedie DJ, et al. Membrane transporters in drug development. Nat Rev Drug Discov. 2010;9:215-36.

6. US Department of Health and Human Services Food and Drug Administration. Guidance for industry drug interaction studies study design, data analysis, implications for dosing, and labeling recommendation. 2012. http://www.fda.gov/downloads/drugs/ guidancecomplianceregulatoryinformation/guidances/ ucm292362.pdf. Accessed 28 Jan 2015.

7. Giacomini KM, Huang SM. Transporters in drug development and clinical pharmacology. Clin Pharmacol Ther. 2013;94:3-9.

8. European Medicines Agency. Guideline on the investigation of drug interaction. http://www.ema.europa.eu/docs/en_GB/docu ment_library/Scientific_guideline/2012/07/WC500129606.pdf. Accessed 28 Jan 2015.

9. Ministry of Health, Labour and Welfare. 2014. http://www.nihs. go.jp/mhlw/20131488.pdf. Accessed 28 Jan 2015.

10. Fojo AT, Ueda K, Slamon DJ, et al. Expression of a multidrugresistance gene in human tumors and tissues. Proc Natl Acad Sci USA. 1987;84:265-9.

11. Lown KS, Mayo RR, Leichtman AB, et al. Role of intestinal P-glycoprotein (mdr1) in interpatient variation in the oral bioavailability of cyclosporine. Clin Pharmacol Ther. 1997;62:248-60.

12. Hashida T, Masuda S, Uemoto S, et al. Pharmacokinetic and prognostic significance of intestinal MDR1 expression in recipients of living-donor liver transplantation. Clin Pharmacol Ther. 2001;69:308-16.

13. Ieiri I. Functional significance of genetic polymorphisms in P-glycoprotein (MDR1, ABCB1) and breast cancer resistance protein (BCRP, ABCG2). Drug Metab Pharmacokinet. 2012;27:85-105.

14. Chowbay B, Li H, David M, et al. Meta-analysis of the influence of MDR1 C3435T polymorphism on digoxin pharmacokinetics and MDR1 gene expression. $\mathrm{Br} \mathrm{J}$ Clin Pharmacol. 2005;60:159-71.

15. Van Limbergen J, Russell RK, Nimmo ER, et al. Genetics of the innate immune response in inflammatory bowel disease. Inflamm Bowel Dis. 2007;13:338-55. 
16. Zintzaras E. Is there evidence to claim or deny association between variants of the multidrug resistance gene (MDR1 or $\mathrm{ABCB} 1)$ and inflammatory bowel disease? Inflamm Bowel Dis. 2012;18:562-72.

17. Doyle LA, Yang W, Abruzzo LV, et al. A multidrug resistance transporter from human MCF-7 breast cancer cells. Proc Natl Acad Sci USA. 1998;95:15665-70.

18. Krishnamurthy P, Schuetz JD. Role of ABCG2/BCRP in biology and medicine. Annu Rev Pharmacol Toxicol. 2006;46:381-410.

19. Jonker JW, Buitelaar M, Wagenaar E, et al. The breast cancer resistance protein protects against a major chlorophyll-derived dietary phototoxin and protoporphyria. Proc Natl Acad Sci USA. 2002;99:15649-54.

20. van Herwaarden AE, Wagenaar E, Merino G, et al. Multidrug transporter $\mathrm{ABCG} /$ breast cancer resistance protein secretes riboflavin (vitamin B2) into milk. Mol Cell Biol. 2007;27:1247-53.

21. Takada T, Ichida K, Matsuo H, et al. ABCG2 dysfunction increases serum uric acid by decreased intestinal urate excretion. Nucl Nucleot Nucl Acids. 2014;33:275-81.

22. Robey RW, To KK, Polgar O, et al. ABCG2: a perspective. Adv Drug Deliv Rev. 2009;61:3-13.

23. Polgar O, Robey RW, Bates SE. ABCG2: structure, function and role in drug response. Expert Opin Drug Metab Toxicol. 2008:4:1-15.

24. Furukawa T, Wakabayashi K, Tamura A, et al. Major SNP (Q141 K) variant of human $\mathrm{ABC}$ transporter $\mathrm{ABCG} 2$ undergoes lysosomal and proteasomal degradations. Pharm Res. 2009;26:469-79.

25. Yang Q, Köttgen A, Dehghan A, et al. Multiple genetic loci influence serum urate levels and their relationship with gout and cardiovascular disease risk factors. Circ Cardiovasc Genet. 2010;3:523-30.

26. Dehghan A, Köttgen A, Yang Q, et al. Association of three genetic loci with uric acid concentration and risk of gout: a genomewide association study. Lancet. 2008;372:1953-61.

27. Gerloff T, Stieger B, Hagenbuch B, et al. The sister of P-glycoprotein represents the canalicular bile salt export pump of mammalian liver. J Biol Chem. 1998;273:10046-50.

28. Kubitz R, Dröge C, Stindt J, et al. The bile salt export pump (BSEP) in health and disease. Clin Res Hepatol Gastroenterol. 2012;36:536-53.

29. US Department of Health and Human Services Food and Drug Administration. Guidance for industry drug-induced liver injury: premarketing clinical evaluation. 2009. http://www.fda.gov/ downloads/Drugs/.../Guidances/UCM174090.pdf. Accessed 28 Jan 2015.

30. Hillgren KM, Keppler D, Zur AA, et al. Emerging transporters of clinical importance: an update from the International Transporter Consortium. Clin Pharmacol Ther. 2013;94:52-63.

31. Keppler D. Multidrug resistance proteins (MRPs, ABCCs): importance for pathophysiology and drug therapy. Handb Exp Pharmacol. 2011:299-323.

32. Suzuki H, Sugiyama Y. Excretion of GSSG and glutathione conjugates mediated by MRP1 and cMOAT/MRP2. Semin Liver Dis. 1998;18:359-76.

33. Nies AT, Keppler D. The apical conjugate efflux pump ABCC2 (MRP2). Pflugers Arch. 2007;453:643-59.

34. Kartenbeck J, Leuschner U, Mayer R, et al. Absence of the canalicular isoform of the MRP gene-encoded conjugate export pump from the hepatocytes in Dubin-Johnson syndrome. Hepatology. 1996;23:1061-6.

35. Keppler D. The roles of MRP2, MRP3, OATP1B1, and OATP1B3 in conjugated hyperbilirubinemia. Drug Metab Dispos. 2014;42:561-5.

36. Scheffer GL, Kool M, de Haas M, et al. Tissue distribution and induction of human multidrug resistant protein 3. Lab Invest. 2002;82:193-201.
37. Hirohashi T, Suzuki H, Sugiyama Y. Characterization of the transport properties of cloned rat multidrug resistance-associated protein 3 (MRP3). J Biol Chem. 1999;274:15181-5.

38. Hirohashi T, Suzuki H, Takikawa $\mathrm{H}$, et al. ATP-dependent transport of bile salts by rat multidrug resistance-associated protein 3 (Mrp3). J Biol Chem. 2000;275:2905-10.

39. Donner MG, Keppler D. Up-regulation of basolateral multidrug resistance protein 3 (Mrp3) in cholestatic rat liver. Hepatology. 2001;34:351-9.

40. Deeley RG, Westlake C, Cole SP. Transmembrane transport of endo- and xenobiotics by mammalian ATP-binding cassette multidrug resistance proteins. Physiol Rev. 2006;86:849-99.

41. Zelcer N, van de Wetering $\mathrm{K}$, de Waart R, et al. Mice lacking Mrp3 (Abcc3) have normal bile salt transport, but altered hepatic transport of endogenous glucuronides. J Hepatol. 2006;44:768-75.

42. Li C, Krishnamurthy PC, Penmatsa H, et al. Spatiotemporal coupling of cAMP transporter to CFTR chloride channel function in the gut epithelia. Cell. 2007;131:940-51.

43. Ritter CA, Jedlitschky G, Meyer zu Schwabedissen H, et al. Cellular export of drugs and signaling molecules by the ATPbinding cassette transporters MRP4 (ABCC4) and MRP5 (ABCC5). Drug Metab Rev. 2005;37:253-78.

44. Rius M, Nies AT, Hummel-Eisenbeiss J, Jedlitschky G, Keppler D. Cotransport of reduced glutathione with bile salts by MRP4 (ABCC4) localized to the basolateral hepatocyte membrane. Hepatology. 2003;38:374-84.

45. Gradhand U, Lang T, Schaeffeler E, et al. Variability in human hepatic MRP4 expression: influence of cholestasis and genotype. Pharmacogenomics J. 2008;8:42-52.

46. Tanaka Y, Manabe A, Fukushima $\mathrm{H}$, et al. Multidrug resistance protein 4 (MRP4) polymorphisms impact the 6-mercaptopurine dose tolerance during maintenance therapy in Japanese childhood acute lymphoblastic leukemia. Pharmacogenomics J. 2014.

47. Ban $\mathrm{H}$, Andoh $\mathrm{A}$, Imaeda $\mathrm{H}$, et al. The multidrug-resistance protein 4 polymorphism is a new factor accounting for thiopurine sensitivity in Japanese patients with inflammatory bowel disease. J Gastroenterol 2010;45:1014-21.

48. Jacquemin E, Hagenbuch B, Stieger B, et al. Expression cloning of a rat liver $\mathrm{Na}(+)$-independent organic anion transporter. Proc Natl Acad Sci USA. 1994;91:133-7.

49. Hagenbuch B, Meier PJ. The superfamily of organic anion transporting polypeptides. Biochim Biophys Acta. 2003;1609:1-18.

50. Tamai I, Nezu J, Uchino H, et al. Molecular identification and characterization of novel members of the human organic anion transporter (OATP) family. Biochem Biophys Res Commun. 2000;273:251-60.

51. Niemi M, Pasanen MK, Neuvonen PJ. Organic anion transporting polypeptide 1B1: a genetically polymorphic transporter of major importance for hepatic drug uptake. Pharmacol Rev. 2011;63:157-81.

52. Ichimaru N, Takahara S, Kokado $\mathrm{Y}$, et al. Changes in lipid metabolism and effect of simvastatin in renal transplant recipients induced by cyclosporine or tacrolimus. Atherosclerosis. 2001;158:417-23.

53. Elsby R, Hilgendorf C, Fenner K. Understanding the critical disposition pathways of statins to assess drug-drug interaction risk during drug development: it's not just about OATP1B1. Clin Pharmacol Ther. 2012;92:584-98.

54. Tirona RG, Leake BF, Merino G, et al. Polymorphisms in OATP$\mathrm{C}$ : identification of multiple allelic variants associated with altered transport activity among European- and African-Americans. J Biol Chem. 2001;276:35669-75.

55. Yoshida K, Maeda K, Sugiyama Y. Hepatic and intestinal drug transporters: prediction of pharmacokinetic effects caused by 
drug-drug interactions and genetic polymorphisms. Annu Rev Pharmacol Toxicol. 2013;53:581-612.

56. Terada $\mathrm{T}$, Inui $\mathrm{K}$. Peptide transporters: structure, function, regulation and application for drug delivery. Curr Drug Metab. 2004;5:85-94.

57. Sawada K, Terada T, Saito H, et al. Recognition of L-amino acid ester compounds by rat peptide transporters PEPT1 and PEPT2. J Pharmacol Exp Therap. 1999;291:705-9.

58. Sugawara M, Huang W, Fei YJ, et al. Transport of valganciclovir, a ganciclovir prodrug, via peptide transporters PEPT1 and PEPT2. J Pharm Sci. 2000;89:781-9.

59. Merlin D, Si-Tahar M, Sitaraman SV, et al. Colonic epithelial hPepT1 expression occurs in inflammatory bowel disease: transport of bacterial peptides influences expression of MHC class 1 molecules. Gastroenterology. 2001;120:1666-79.

60. Wojtal KA, Eloranta JJ, Hruz P, et al. Changes in mRNA expression levels of solute carrier transporters in inflammatory bowel disease patients. Drug Metab Dispos. 2009;37:1871-7.

61. Merlin D, Steel A, Gewirtz AT, et al. hPepT1-mediated epithelial transport of bacteria-derived chemotactic peptides enhances neutrophil-epithelial interactions. J Clin Investig. 1998;102:2011-8.

62. Vavricka SR, Musch MW, Chang JE, et al. hPepT1 transports muramyl dipeptide, activating NF-kappaB and stimulating IL-8 secretion in human colonic Caco2/bbe cells. Gastroenterology. 2004;127:1401-9.

63. Dalmasso G, Nguyen HT, Ingersoll SA, et al. The PepT1-NOD2 signaling pathway aggravates induced colitis in mice. Gastroenterology. 2011;141:1334-45.

64. Ingersoll SA, Ayyadurai S, Charania MA, et al. The role and pathophysiological relevance of membrane transporter PepT1 in intestinal inflammation and inflammatory bowel disease. Am J Physiol Gastrointest Liver Physiol. 2012;302:G484-92.

65. Giacomini KM, Balimane PV, Cho SK, et al. International Transporter Consortium commentary on clinically important transporter polymorphisms. Clin Pharmacol Ther. 2013;94:23-6.

66. Pasanen MK, Neuvonen M, Neuvonen PJ, et al. SLCO1B1 polymorphism markedly affects the pharmacokinetics of simvastatin acid. Pharm Genom. 2006;16:873-9.

67. Link E, Parish S, Armitage J, et al. SLCO1B1 variants and statininduced myopathy-a genomewide study. $\mathrm{N}$ Engl $\mathrm{J}$ Med. 2008;359:789-99.

68. Kajosaari LI, Niemi M, Neuvonen M, et al. Cyclosporine markedly raises the plasma concentrations of repaglinide. Clin Pharmacol Ther. 2005;78:388-99.

69. Treviño LR, Shimasaki N, Yang W, et al. Germline genetic variation in an organic anion transporter polypeptide associated with methotrexate pharmacokinetics and clinical effects. J Clin Oncol. 2009;27:5972-8.

70. D'Avolio A, Carcieri C, Cusato J, et al. Intracellular accumulation of atazanavir/ritonavir according to plasma concentrations and OATP1B1, ABCB1 and PXR genetic polymorphisms. J Antimicrob Chemother. 2014;69:3061-6.

71. Kohlrausch FB, de Cássia Estrela R, Barroso PF, et al. The impact of SLCO1B1 polymorphisms on the plasma concentration of lopinavir and ritonavir in HIV-infected men. Br J Clin Pharmacol. 2010;69:95-8.

72. Adkison KK, Vaidya SS, Lee DY, et al. Oral sulfasalazine as a clinical BCRP probe substrate: pharmacokinetic effects of genetic variation (C421A) and pantoprazole coadministration. J Pharm Sci. 2010;99:1046-62.

73. Urquhart BL, Ware JA, Tirona RG, et al. Breast cancer resistance protein (ABCG2) and drug disposition: intestinal expression, polymorphisms and sulfasalazine as an in vivo probe. Pharma Genom. 2008;18:439-48.

74. Yamasaki Y, Ieiri I, Kusuhara H, et al. Pharmacogenetic characterization of sulfasalazine disposition based on NAT2 and ABCG2 (BCRP) gene polymorphisms in humans. Clin Pharmacol Ther. 2008;84:95-103.

75. Zhang W, Yu BN, He YJ, et al. Role of BCRP 421C $>$ A polymorphism on rosuvastatin pharmacokinetics in healthy Chinese males. Clin Chim Acta. 2006;373:99-103.

76. Bailey KM, Romaine SP, Jackson BM, et al. Hepatic metabolism and transporter gene variants enhance response to rosuvastatin in patients with acute myocardial infarction: the GEOSTAT-1 Study. Circ Cardiovasc Genet. 2010;3:276-85.

77. Tomlinson $\mathrm{B}, \mathrm{Hu} \mathrm{M}$, Lee $\mathrm{VW}$, et al. ABCG2 polymorphism is associated with the low-density lipoprotein cholesterol response to rosuvastatin. Clin Pharmacol Ther. 2010;87:558-62.

78. Keskitalo JE, Zolk O, Fromm MF, et al. ABCG2 polymorphism markedly affects the pharmacokinetics of atorvastatin and rosuvastatin. Clin Pharmacol Ther. 2009;86:197-203.

79. Keskitalo JE, Pasanen MK, Neuvonen PJ, et al. Different effects of the $\mathrm{ABCG} 2 \mathrm{c} .421 \mathrm{C}>\mathrm{A}$ SNP on the pharmacokinetics of fluvastatin, pravastatin and simvastatin. Pharmacogenomics. 2009;10:1617-24.

80. Takahashi N, Miura M. Therapeutic drug monitoring of imatinib for chronic myeloid leukemia patients in the chronic phase. Pharmacology. 2011;87:241-8.

81. Cusatis G, Gregorc V, Li J, et al. Pharmacogenetics of ABCG2 and adverse reactions to gefitinib. $\mathrm{J}$ Natl Cancer Inst. 2006;98:1739-42.

82. Fukudo M, Ikemi Y, Togashi Y, et al. Population pharmacokinetics/pharmacodynamics of erlotinib and pharmacogenomic analysis of plasma and cerebrospinal fluid drug concentrations in Japanese patients with non-small cell lung cancer. Clin Pharmacokinet. 2013;52:593-609.

83. Thomas F, Rochaix P, White-Koning M, et al. Population pharmacokinetics of erlotinib and its pharmacokinetic/pharmacodynamic relationships in head and neck squamous cell carcinoma. Eur J Cancer. 2009;45:2316-23.

84. Mizuno T, Terada T, Kamba T, et al. ABCG2 421C > A polymorphism and high exposure of sunitinib in a patient with renal cell carcinoma. Ann Oncol. 2010;21:1382-3.

85. Mizuno T, Fukudo M, Terada T, et al. Impact of genetic variation in breast cancer resistance protein (BCRP/ABCG2) on sunitinib pharmacokinetics. Drug Metab Pharmacokinet. 2012;27:631-9.

86. Mizuno T, Fukudo M, Fukuda T, et al. The effect of ABCG2 genotype on the population pharmacokinetics of sunitinib in patients with renal cell carcinoma. Ther Drug Monit. 2014;36:310-6. 